GESTIÓN Y

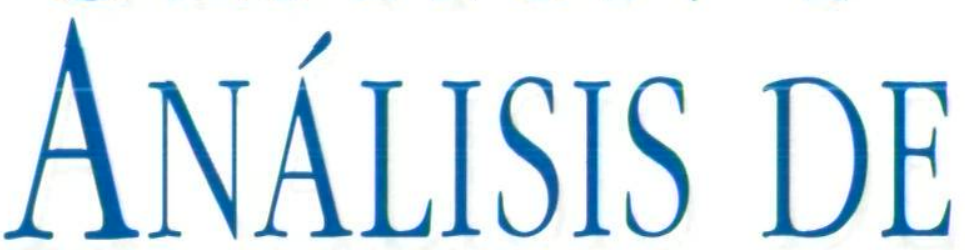

POLÍTICAS PÚBLICAS

EVALUACIÓN Y CALIDAD DE LAS POLÍTICAS PÚBLICAS
Presentación. Juan Manuel García Falcón.

Calidad y cantidad en el análisis de las politicas públicas. Geert Bouckaert.

Una comparación internacional en la evaluación de las politicas públicas. Hans-Ulrich Derlien.

El surgimiento, declive y resurgimiento de la evaluación de políticas públicas en el Gobierno americano. Guy B. Peters.

La gestión de la calidad en la Administración General del Estado. Amador Elena Córdoba.

Gobernar es gestionar con calidad. Jordi López Camps.

La integración de la evaluación de políticas públicas en el proceso presupuestario. Eduardo Zapico Goñi.

Implantación del Plan de Calidad en la Comunidad de Madrid (1995-1998). José Luis Moreno Casas.

La mejora continua de la calidad en la Universidad: algunas experiencias en la Universidad Carlos III de Madrid. Daniel Peña.

La calidad como yacimiento de la nueva gestión pública: la experiencia del Instituto Nacional de la Seguridad Social. Vicente Pérez Menayo.

El rol del evaluador: textos para un debate.

Deontología de la evaluación: el modelo de los códigos éticos anglosajones. María Bustelo Ruesta.

Informe sobre evaluación de la calidad en las Universidades. Consejo de Universidades.
Otros APARTADOS

\section{AMÉRICA LATINA.}

REFORMAR EL ESTADO.

BIBLIOGRAFÍA SOBRE EL TEMA. 
GESTIÓN Y

ANÁLISIS DE

POLÍTICAS PÚBLICAS

Revista coeditada por el Instituto Nacional de Administración Pública, el Instituto de Estudios Fiscales, la Escuela de Organización Industrial y el Boletín Oficial del Estado

\section{CONSEJO DE REDACCIÓN}

Presidente: D. Enrique Álvarez Conde. Director del INAP. Vicepresidentes: D. Julio Seage Mariño, Director General del Boletín Oficial del Estado; D. Jesús Bermejo Ramos, Director General del Instituto de Estudios Fiscales; D. Félix Santamaría Díez, Director de la Fundación "Escuela de Organización Industrial". Vocales: D. Manuel Arenilla Sáez, D. Mariano Baena del Alcázar, D. Ernesto Carrillo Barroso, D. Rafael Catalá Polo, D. Koldo Echebarría Ariznabarreta, D. Amador Elena Córdoba, D. Juan Manuel García Falcón, D. Juan Carlos González Hernández, D. Vicente González Radio, D. Luis González Seara, D. Enrique Martín López, D. Eugenio Nasarre Goicoechea, D. Alberto Nuñez Feijóo, Dª Blanca Olías de Lima, D. Manuel Ortigueira Bouzada, Dª Ana María Pastor Julián, D. Francisco Vanaclocha Bellver, D. José Vilas Nogueira, D. Eduardo Zapico Goñi.

\section{DIRECTOR}

D. Carlos-Ramiro Alba Tercedor

\section{SECRETARIO}

D. José Manuel Canales Aliende

\section{Edita:}

INSTITUTO NACIONAL DE ADMINISTRACIÓN PĹBLICA

NIPO: $329-98-005-5$

ISSN: $1134-6035$

DEPÓSITO LEGAL: M. 39.657-1994

Fotocomposición e impresión:

Solana e hijos A. G., S. A.

San Alfonso,26 La Fortuna-Leganés

28917 Madrid

\section{Distribución y suscripciones:}

INSTITLTO NACIONAL DE ADMINISTRACIÓN PĹBLICA CENTRO DE PLBLICACIONES

Atocha, 106. 28012 MADRID

Teléfono: $3493211 \quad$ Fax: 3493287

\section{Precios:}

Nümero suelto

Número doble

Suscripción anual

( 3 núms. año)
$1.500 \mathrm{pts}$.

3.000 pts

+.000 pts.

IVA INCLUIDO 


\section{Sumario}

EvaLUACIÓN Y CALIDAD DE LAS POLÍTICAS PÚBLICAS
Presentación. Juan Manuel García Falcón.

$\underline{\text { Página }}$

Calidad y cantidad en el análisis de las políticas públicas. Geert Bouckaert.

Una comparación internacional en la evahuación de las politicas püblicas. Hans-Ulrich Derlien.

El surgimiento, declive y resurgimiento de la evaluación de politicas publicas en el Gobierno americano. Guy B. Peters.

La gestión de la calidad en la Administración General del Estado. Amador Elena Córdoba.

Gobernar es gestionar con calidad. Jordi López Camps.

Ia integración de la evaluación de políticas públicas en el proceso presupuestario. Eduardo Zapico Goñi.

EXPERIENCIAS Y CASOS

Implantación del Plan de Calidad en la Comunidad de Madrid (1995-1998). José Luis Moreno Casas.

La mejora continua de la calidad en la Universidad: algunas experiencias en la Universidad Carlos III de Madrid. Daniel Peña.

La calidad como yacimiento de la nueva gestión pública: la experiencia del Instituto Nacional de la Seguridad Social. Vicente Pérez Menayo.

El rol del evaluador: textos para un debate.

Otras secciones

Deontología de la evaluación: el modelo de los códigos éticos anglosajones. María Bustelo Ruesta.

Informe sobre evaluación de la calidad de las Universidades. Consejo de Universidades.

América latina

Evaluación organizacional del Programa de Modernización Administrativa del D.D.F. David Arellano Gault - Colaboración: Jorge

E. Culebro Moreno y Miguel A. Gutiérrez.

Sistema municipal de gestión de calidad. La aplicación de los modelos de calidad a la gestión pública. Antonio Díaz.

La uindustrian de la evaluación y bibliografia básica. Xavier Ballart. 231 


\section{Normas para la presentación de originales}

1. Los manuscritos se aceptarán considerando que contienen trabajos no publicados ni presentados para su publicación en otros medios, y que todas las personas que aparecen como autores han dado su aprobación para que los trabajos se presenten a la revista.

2. Todos los manuscritos se presentarán para su evaluación, y podrán ser aceptados para su publicación, sujetos a revisión o rechazados.

3. Los manuscritos deberán presentarse atendiendo a las siguientes normas:

a) Se presentarán en soporte magnético e impresos a doble espacio. En la primera página deberá constar el tínulo del trabajo, nombre del autor/es e institución a la que pertenecen, así como una dirección, y fax en su caso, a la que se enviará noticia de la recepción del trabajo y la correspondencia que pueda originarse.

b) Las notas al texto deberán ir numeradas correlativamente. Sus referencias aparecerán entre paréntesis en el punto del texto en que se requieran y las notas deberán mecanografiarse al final del trabajo, antes de las referencias bibliográficas, bajo la inscripción NOTAS.

c) Las fórmulas matemáticas se enumerarán, en su caso, a la derecha de las mismas. Los cuadros y gráficos deberán presentarse con calidad suficiente para su impresión y, de ser posible, el original, indicándose, en todo caso, sus fuentes.

d) Las referencias bibliográficas deberán aparecer al final del artículo, bajo la inscripción de BIBLIOGRAFÍA, por orden alfabético de autores $y$, en cada una, adaptándose al siguiente orden: Autor, año de publicación (distinguiendo a, b, c, si hay varias que corresponden al mismo autor y año), título del artículo o libro, título de la revista o editorial, lugar de publicación (sólo libros), número de la revista y páginas. Cuando se citen en el texto, se usará de este modo (LÓPEZ, 1990), LOPEZ (1990), (LÓPEZ, GÓMEZ y GARCÍA, 1990) o (LÓPEZ et al., 1990) cuando sean más de tres. e) Las palabras que se desee aparezcan en cursiva, deberían subrayarse en el original.

4. Los manuscritos de los artículos, estudios o aportaciones doctrinales que se remitan para su evaluación y, en su caso, posterior publicación, tendrán una extensión mínima indicativa de quince páginas o folios mecanografiados.

Junto a los mismos deberá acompañarse, además, un resumen breve de ellos, de no más de cien palabras, más un abstracto en inglés de la mișma longitud.

5. Los manuscritos de las aportaciones al resto de los apartados del Sumario de la Revista, deberán adaptarse a la extensión mínima y máxima que de forma indicativa serían:

a) “Experiencias»: una extensión máxima de veinte páginas.

b) “Documentación: una extensión máxima de treinta páginas.

c) "Recensiones bibliográficas": una extensión entre cinco y diez páginas.

Además, los manuscritos con aportaciones doctrinales, experiencias, documentación y recensiones bibliográficas, podrán también enviarse por particulares o por las Entidades e Instituciones interesadas, cualquier noticia de actualidad o evento que consideren que es de utilidad para su inclusión, en su caso, por el Consejo de Redacción de la Revista, dentro del apartado ‘Crónica y Agendan.

7. Los artículos y demás colaboraciones para ser evaluados por el Consejo de Redacción de la Revista y, en su caso, publicados se enviarán a:

Secretaŕa de la Revista «Gestión y Análisis de Políticas Públicas"

Centro de Publicaciones

INAP

Calle Atocha, 106

28012 MADRID

Teléfono: (91) 3493119

FAX: (91) 3493287 